\title{
A SPLITTING THEOREM FOR HIGHER ORDER PARALLEL IMMERSIONS
}

\author{
INES KATH AND PAUL-ANDI NAGY \\ (Communicated by Ken Ono)
}

\begin{abstract}
We consider isometric immersions into space forms having the second fundamental form parallel at order $k$. We show that this class of immersions consists of local products, in a suitably defined sense, of parallel immersions and normally flat immersions of flat spaces.
\end{abstract}

\section{INTRODUCTION}

A basic question in submanifold geometry is the study of isometric immersions of Riemannian manifolds having their second fundamental form subject to certain geometric or analytic properties. One of the best known examples is the class of immersions having parallel second fundamental form. Such immersions are called parallel. Their study is part of well-established theories; for instance, the Euclidean case has been fully described by Ferus [5, 6, 7. Parallel immersions in space forms are the extrinsic counterpart of locally symmetric spaces [21].

In this spirit, a natural class to consider is that of $k$-parallel immersions, by requiring the second fundamental form to be parallel at order $k$ for $k \geqslant 1$. At a pure analogy level, while each Riemannian manifold whose Riemannian curvature tensor is parallel at higher order is locally symmetric [20 the class of $k$-parallel immersions is sensibly larger than that of parallel ones. Curves such as the Cornu spiral provide simple 2-parallel, nonparallel examples.

Early work by Mirzojan [15] on $k$-parallel immersions has been taken up by Dillen and Lumiste among others; see [14] for an overview. In the case of immersions into space forms, classification results have been obtained for low dimension or codimension or for small $k$ in $[9,2,2,12,10,11,13$. A case-by-case inspection of instances therein reveals that the immersion is a product and each factor is either a $k$-parallel curve, a $k$-parallel flat and normally flat surface, an affine subspace or a sphere.

That this reflects the general structure of $k$-parallel immersions into space forms is the main result of this paper. We prove

Theorem. Any k-parallel isometric immersion of a Riemannian manifold $(M, g)$ into a simply connected space form is the local product of a parallel immersion and a k-parallel immersion of a flat space with flat normal bundle.

Received by the editors March 14, 2011.

2010 Mathematics Subject Classification. Primary 53B21, 53C42.

Key words and phrases. Submanifold, higher order parallel fundamental form, splitting theorems. 
This is Theorem 2.3 in the paper. Products of immersions are understood in the sense of Nölker 19]; see Definition 2.4. Open manifolds are naturally considered, since in the compact case integration shows that $k$-parallel immersions are parallel.

To prove the theorem we first note that having the ambient space locally symmetric forces the Riemann curvature to be parallel at higher order, hence parallel by 20. We refine the natural local product decomposition of $(M, g)$ into a flat space and a locally symmetric space with nondegenerate curvature. The key ingredient is to take into account the algebraic structure of the normal bundle reflecting the structure of higher order derivatives of the second fundamental form. It is used to modify the above-mentioned splitting into one that satisfies the requirements in the generalised Moore splitting criterion [16, 19].

Our theorem reduces the study of $k$-parallel immersions into space forms to that of parallel immersions and $k$-parallel normally flat immersions of flat space. This is an essential step towards the classification of $k$-parallel immersions of space forms. Indeed, parallel immersions of space forms are known. The Euclidean case was solved by Ferus as already mentioned above. If the target space is a sphere one can easily get a classification from the Euclidean case. For the hyperboloid as target a classification was achieved independently by Takeuchi [22] and Backes and Reckziegel [1. Furthermore, in [13] Lumiste describes a general method (the so-called polynomial map method) for the investigation of $k$-parallel immersions in Euclidean spaces that are flat and normally flat; see also [18, where the latter are interpreted as certain integrable systems of hydrodynamic type. In conclusion, to obtain a full classification there remains to understand the structure of flat, normally flat $k$-parallel immersions in hyperbolic space.

\section{Structure Results}

Let $\left(N^{n}, g\right)$ be a Riemannian manifold and let us consider an isometric immersion $\left(M^{m}, g\right) \hookrightarrow(N, g), m \leqslant n$. We will denote by $\nabla$ and $\nabla^{N}$, respectively, the Levi-Civita connections and by $R, R^{N}$ the Riemann curvature tensors, with the convention that $R^{N}(X, Y, Z, U)=g\left(\left(\nabla^{N}\right)_{Y, X}^{2} Z-\left(\nabla^{N}\right)_{X, Y}^{2} Z, U\right)$ for all $X, Y, Z, U$ in $T N$. The orthogonal splitting

$$
T N=T M \oplus N M
$$

along $M$ enables us to consider the second fundamental form $\alpha: T M \times T M \rightarrow N M$, where $N M$ is the normal bundle of $M$. We denote by $\nabla^{\perp}$ the induced covariant derivative in $N M$ and by $R^{\perp}$ the corresponding curvature tensor. Furthermore, we write $\nabla \alpha$ for the covariant derivative of $\alpha$, which is defined by $\nabla$ and $\nabla^{\perp}$.

Definition 2.1. Let $(M, g) \hookrightarrow(N, h)$ be an isometric immersion. It is called $k$-parallel for some $k \geqslant 1$ if and only if

$$
\nabla^{k} \alpha=0
$$

A particular case thereof consists in the so-called parallel immersions, when one requires $\nabla \alpha=0$.

In most of what follows we will look at immersions into standard spaces $M^{n}(c)$, that is, simply connected, complete spaces of constant sectional curvature $c$ and dimension $n$, realised by their standard models in $\mathbb{R}^{n+1}$ : the sphere, the hyperboloid and the hyperplane. 
The main goal of this paper is to prove that the study of $k$-parallel immersions into standard spaces reduces to that of parallel immersions and $k$-parallel immersions of flat space. The latter class will be shown later on to be normally flat as well.

One of our main tools is Moore's decomposition criterion [17 and its generalisations to the case of standard spaces due to [16] (see also [19]). Its application requires setting up the notion of product immersion, which we recall below in the Euclidean case to begin with.

Definition 2.2. An isometric immersion $f: M_{1} \times M_{2} \rightarrow \mathbb{R}^{n}$ of a Riemannian product is called a product immersion if and only if $f=F \circ\left(f_{1} \times f_{2}\right)$ for isometric immersions $f_{i}: M_{i} \rightarrow N_{i}, i=1,2$, where $N_{1}, N_{2}$ are affine subspaces of $\mathbb{R}^{n}$ such that $F: N_{1} \times N_{2} \rightarrow \mathbb{R}^{n}$ is isometric.

The next definition captures necessary conditions for an immersion to be a product. In the rest of this paper we will systematically use the natural identification $T\left(M_{1} \times M_{2}\right)=T M_{1} \oplus T M_{2}$ whenever $M_{1}, M_{2}$ are smooth manifolds.

Definition 2.3. The second fundamental form $\alpha$ of an isometric immersion $f$ : $M_{1} \times M_{2} \rightarrow M$ is called decomposable if $\alpha\left(\mathscr{D}_{1}, \mathscr{D}_{2}\right)=0$, where $\mathscr{D}_{i}=d f\left(T M_{i}\right), i=$ 1,2 .

Having decomposable second fundamental form has been shown, for Euclidean target spaces, to yield a product structure in [17]; explicitly:

Theorem 2.1. An isometric immersion $f: M_{1} \times M_{2} \rightarrow \mathbb{R}^{n}$, where $M_{1}, M_{2}$ are connected and $f$ has decomposable second fundamental form, is a product immersion.

To present the generalisation of the above result to a standard space target, we first recall that a submanifold $N$ in $M^{n}(c)$ is called spherical if its second fundamental form $\alpha=g \otimes \zeta$ for some parallel normal vector field, where $g$ is the induced metric on $N$. For $c \neq 0$ this is equivalent to $N$ being the intersection of the standard space and some affine subspace of $\mathbb{R}^{n+1}$.

Definition 2.4. An isometric immersion $f: M_{1} \times M_{2} \rightarrow M^{n}(c)$ of a Riemannian product is called a product immersion if and only if $f=F \circ\left(f_{1} \times f_{2}\right)$ for isometric immersions $f_{i}: M_{i} \rightarrow N_{i}, i=1,2$, where:

(i) $N_{1}, N_{2}$ are isometric to standard spaces and admit isometric embeddings $\varphi_{i}: N_{i} \rightarrow M^{n}(c), i=1,2$, with spherical image such that

$$
\varphi_{1}\left(N_{1}\right) \cap \varphi_{2}\left(N_{2}\right)=\{\bar{p}\} ;
$$

(ii) the map $F: N_{1} \times N_{2} \rightarrow \mathbb{R}^{n+1},\left(p_{1}, p_{2}\right) \mapsto \bar{p}+\left(\varphi_{1}\left(p_{1}\right)-\bar{p}\right)+\left(\varphi_{2}\left(p_{2}\right)-\bar{p}\right)$ is an isometric embedding with image contained in $M^{n}(c)$.

The curved counterpart of Moore's decomposition criterion in Theorem 2.1 is

Theorem 2.2 ([16]; see also [19]). An isometric immersion $f: M_{1} \times M_{2} \rightarrow M^{n}(c)$, where $M_{1}, M_{2}$ are connected and $f$ has decomposable second fundamental form, is a product immersion.

Whereas Definition 2.2 is a particular case of Definition 2.4 the latter allows a broader treatment of the case when $c=0$, e.g. when $N_{1}, N_{2}$ are taken to be spheres. 
An isometric immersion $f: M \rightarrow N$ will be called a local product if for each $x$ in $M$ there exists a product open set $M_{1} \times M_{2}$ around $x$ such that $f_{\mid M_{1} \times M_{2}}$ is a product immersion.

The main result in this paper can now be formulated as follows.

Theorem 2.3. Let $(M, g) \rightarrow M^{n}(c)$ be a $k$-parallel isometric immersion. It is the local product of a parallel immersion and a $k$-parallel immersion of a flat space with flat normal bundle. When $(M, g)$ is simply connected and complete, the splitting is global.

The proof requires a few technical steps, which we will outline below. When not specified otherwise we will work under the assumptions of Theorem 2.3 .

We define at each point of $M$,

$$
E_{0}=\{X \in T M: R(T M, T M) X=0\}, E_{1}=E_{0}^{\perp},
$$

and note that $E_{1}=\operatorname{span}\{R(X, Y) Z: X, Y, Z \in T M\}$.

Proposition 2.1. Let $(M, g) \hookrightarrow(N, h)$ be a $k$-parallel isometric immersion, where $(N, h)$ is locally symmetric. The following hold:

(i) $E_{0}$ and $E_{1}$ are parallel in $T M$;

(ii) the distribution $E_{0}$ is flat;

(iii) $E_{1} \subset\left\{X \in T M: \nabla_{X} \alpha=0\right\}$.

Proof. (i) We differentiate the Gauss equation

$$
\begin{aligned}
R^{N}\left(X_{1}, X_{2}, X_{3}, X_{4}\right)= & R\left(X_{1}, X_{2}, X_{3}, X_{4}\right)+\left\langle\alpha_{X_{2}} X_{3}, \alpha_{X_{1}} X_{4}\right\rangle \\
& -\left\langle\alpha_{X_{1}} X_{3}, \alpha_{X_{2}} X_{4}\right\rangle,
\end{aligned}
$$

where $X_{i}, 1 \leq i \leq 4$, belong to $T M$, to find that

$$
\left(\nabla^{2 k-1} R\right)\left(X_{1}, X_{2}, X_{3}, X_{4}\right)=\left(\nabla^{2 k-1} R^{N}\right)\left(X_{1}, X_{2}, X_{3}, X_{4}\right)
$$

in directions tangent to $M$. Now, taking into account that $\nabla^{N} R^{N}=0$ yields

$$
-\left(\nabla_{X_{0}} R^{N}\right)\left(X_{1}, X_{2}, X_{3}, X_{4}\right)=\sum_{i=1}^{4} R^{N}\left(X_{1}, \ldots, \alpha_{X_{0}} X_{i}, \ldots, X_{4}\right)
$$

for all $X_{0}$ in $T M$. Now the Codazzi-Mainardi formula

$$
-R^{N}\left(X_{1}, X_{2}, X_{3}, \xi\right)=\left\langle\left(\nabla_{X_{1}} \alpha\right)_{X_{2}} X_{3}-\left(\nabla_{X_{2}} \alpha\right)_{X_{1}} X_{3}, \xi\right\rangle
$$

for $\xi \in N M$ gives, for instance, that

$$
-R^{N}\left(X_{1}, X_{2}, X_{3}, \alpha_{X_{0}} X_{4}\right)=\left\langle\left(\nabla_{X_{1}} \alpha\right)_{X_{2}} X_{3}-\left(\nabla_{X_{2}} \alpha\right)_{X_{1}} X_{3}, \alpha_{X_{0}} X_{4}\right\rangle ;
$$

hence clearly $\nabla^{2 k-1} R^{N}=0$ and then $\nabla^{2 k-1} R=0$. By [20] we have that $\nabla R=0$, whence the claim.

(ii) follows from the definition of $E_{0}$.

(iii) We exploit an argument used in [20, 23] under slightly different assumptions. The function $t=|\alpha|^{2}: M \rightarrow \mathbb{R}$ satisfies

$$
\left\langle\nabla_{X} \operatorname{grad} t, Y\right\rangle=2\left\langle\nabla_{X} \alpha, \nabla_{Y} \alpha\right\rangle
$$

for all $X, Y$ in $T M$; it follows that $\nabla^{2 k-2} \operatorname{grad} t=0$ along $M$, after differentiating at higher order. In particular, $\nabla^{2 k-2} X_{1}=0$, where $X_{1}$ denotes the orthogonal projection of grad $t$ onto the parallel distribution $E_{1}$. Now we can use the following result due to Tanno. Suppose that at some point $x$ of a Riemannian manifold and for some tangent vectors $X, Y$ at $x, R(X, Y)$ is not singular. Then having 
$\nabla^{k} T=0$ for an arbitrary tensor $T$ and for some $k \geq 1$ implies that $\nabla T=0$; see [23], Theorem 1. Applied to the integral manifolds of $E_{1}$ this yields $\nabla_{U} X_{1}=0$ for all $U$ in $E_{1}$; in particular, $R\left(E_{1}, E_{1}\right) X_{1}=0$. Since, moreover, $R\left(E_{0}, T M\right)=0$ we get $R(T M, T M) X_{1}=0$; thus $X_{1} \in E_{0}$ showing that $X_{1}=0$. In other words, gradt belongs to $E_{0}$, which is parallel in $T M$. When taking $X, Y$ in $E_{1}$ it follows that the right-hand side of (2.4) vanishes and the claim follows by a positivity argument.

Under additional assumptions we have the following alternative by using essentially that a Ricci flat, locally symmetric space is flat.

Corollary 2.1. If we have a k-parallel isometric immersion of an Einstein or locally irreducible manifold $M$ into a locally symmetric space, then either the immersion is parallel or $M$ is flat.

In what follows the action of the curvature tensor on a tensor field $Q$ in $\left(T^{\star} M\right)^{l} \otimes$ $N M$ is given by

$$
\begin{aligned}
(R(X, Y) \cdot Q)\left(Z_{1}, \ldots, Z_{l}\right)= & R^{\perp}(X, Y) Q\left(Z_{1}, \ldots, Z_{l}\right) \\
& -\sum_{i=1}^{l} Q\left(Z_{1}, \ldots, R(X, Y) Z_{i}, \ldots, Z_{l}\right)
\end{aligned}
$$

whenever $X, Y, Z_{i}, 1 \leq i \leq l$, belong to $T M$. For $s \geqslant 0$ we will frequently use the shorthand notation $\operatorname{Im} \nabla^{s} \alpha$ to refer to $\operatorname{span}\left\{\nabla_{X_{1}, \ldots, X_{s}}^{s} \alpha\right\} \subseteq N M$, where $X_{i}, 1 \leq$ $i \leqslant s$ belong to $T M$.

Lemma 2.1. Let $(M, g)$ be a k-parallel isometric immersion into a space form. We have

(i) $R^{\perp}\left(E_{0}, T M\right)\left(\operatorname{Im} \nabla^{k-s} \alpha\right)=0, s \geqslant 0$;

(ii) $R^{\perp}\left(E_{0}, T M\right)=0$.

Proof. Because $\nabla^{2}\left(\nabla^{k-2} \alpha\right)=0$ it follows after anti-symmetrisation that the curvature operator $R(X, Y)$ acts trivially on $\nabla^{k-2} \alpha$. Since, moreover, $R\left(E_{0}, T M\right) T M=$ 0 we obtain that

$$
R^{\perp}\left(E_{0}, T M\right)\left(\operatorname{Im} \nabla^{k-2} \alpha\right)=0 .
$$

Further anti-symmetrisation in the first two arguments in $\nabla^{k-2} \alpha=\nabla^{2}\left(\nabla^{k-4} \alpha\right)$ yields

$$
R^{\perp}\left(E_{0}, T M\right)\left(R^{\perp}\left(E_{0}, T M\right)\left(\operatorname{Im} \nabla^{k-4} \alpha\right)\right)=0 .
$$

After taking scalar products with vectors in $\operatorname{Im} \nabla^{k-4} \alpha$ a positivity argument shows that

$$
R^{\perp}\left(E_{0}, T M\right)\left(\operatorname{Im} \nabla^{k-4} \alpha\right)=0 .
$$

Continuing this procedure leads to $R^{\perp}\left(E_{0}, T M\right)\left(\operatorname{Im} \nabla^{k-2 s} \alpha\right)=0, s \geqslant 0$. Since $k$-parallel manifolds are also $(k+1)$-parallel this equation holds also for $k+1$, and (i) follows.

In particular, (i) gives $R^{\perp}\left(E_{0}, T M\right)(\operatorname{Im} \alpha)=0$. We will now use the Ricci formula

$$
\left\langle R^{\perp}(X, Y) \xi_{1}, \xi_{2}\right\rangle=\left\langle\left[A_{\xi_{1}}, A_{\xi_{2}}\right] X, Y\right\rangle
$$

for $X, Y$ in $T M$ and $\xi_{1}, \xi_{2}$ in $N M$, where $\left\langle A_{\xi} X, Y\right\rangle=-\left\langle\alpha_{X} Y, \xi\right\rangle$ is the shape operator. It shows that also $(\operatorname{Im} \alpha)^{\perp}=\left\{\xi \in N M: A_{\xi}=0\right\}$ is annihilated by $R^{\perp}\left(E_{0}, T M\right)$, and (ii) follows. 
Proposition 2.2. Let $(M, g)$ be a $k$-parallel isometric immersion into a space form. Then we have that

(i) $(M, g)$ is semi-parallel; that is, $R(X, Y) \cdot \alpha=0$ whenever $X, Y$ belong to $T M$

(ii) $\nabla R^{\perp}=0$.

Proof. (i) From the lemma and the definition of $E_{0}$ it follows that the tensor

$(R(X, Y) \cdot \alpha)\left(Z_{1}, Z_{2}\right)=R^{\perp}(X, Y)\left(\alpha_{Z_{1}} Z_{2}\right)-\alpha\left(R(X, Y) Z_{1}, Z_{2}\right)-\alpha\left(Z_{1}, R(X, Y) Z_{2}\right)$ vanishes when $X$ is in $E_{0}$. For $X, Y$ in $E_{1}$ we have $\nabla_{X, Y}^{2} \alpha=0$ by (iii) in Proposition 2.1 and $R(X, Y) \cdot \alpha=0$ follows.

(ii) By differentiation in the lemma above,

$$
\left(\nabla_{T M} R^{\perp}\right)\left(E_{0}, T M\right)=0
$$

since $E_{0}$ is parallel inside $T M$. There remains to show that $\left(\nabla_{T M} R^{\perp}\right)\left(E_{1}, E_{1}\right)=0$. For $X, Y$ in $E_{1}, U$ in $T M$ as well as $\xi_{1}, \xi_{2}$ in $N M$, we have

$$
\left\langle\left(\nabla_{U} R^{\perp}\right)(X, Y) \xi_{1}, \xi_{2}\right\rangle=\left\langle\left[\left(\nabla_{U} A\right)_{\xi_{1}}, A_{\xi_{2}}\right] X, Y\right\rangle+\left\langle\left[A_{\xi_{1}},\left(\nabla_{U} A\right)_{\xi_{2}}\right] X, Y\right\rangle
$$

by differentiation in the Ricci equation (2.5). Moreover

$$
\begin{aligned}
\left\langle\left[\left(\nabla_{U} A\right)_{\xi_{1}}, A_{\xi_{2}}\right] X, Y\right\rangle & =\left\langle\left(\nabla_{U} A\right)_{\xi_{1}} A_{\xi_{2}} X, Y\right\rangle-\left\langle A_{\xi_{2}}\left(\nabla_{U} A\right)_{\xi_{1}} X, Y\right\rangle \\
& =\left\langle\left(\nabla_{U} A\right)_{\xi_{1}} Y, A_{\xi_{2}} X\right\rangle-\left\langle A_{\xi_{2}}\left(\nabla_{U} A\right)_{\xi_{1}} X, Y\right\rangle .
\end{aligned}
$$

Now for space forms, the right-hand side in the Codazzi-Mainardi equation (2.3) vanishes. Since, moreover, $\nabla_{E_{1}} A=0$ by (iii) in Proposition 2.1 each summand above is zero. A similar argument shows that the same holds for the second commutator in the expression of $\nabla R^{\perp}$ and the claim is proved.

We consider

$$
N_{0} M=\left\{\xi \in N M: R^{\perp}(T M, T M) \xi=0\right\}
$$

with orthogonal complement $N_{1} M$ in $N M$.

Proposition 2.3. Let $(M, g)$ be a $k$-parallel isometric immersion into a space form. We have:

(i) $N_{0} M, N_{1} M$ are parallel;

(ii) $N_{0} M$ is flat.

Proof. (i) is a direct consequence of (ii) in Proposition 2.2, while (ii) follows from (i) and the definition of $N_{0} M$.

Let us consider the following subbundle of the normal bundle of $M$ :

$$
F_{0}:=\operatorname{span}\left\{\left(\nabla_{X_{1}, \ldots, X_{l}}^{l} \alpha\right)(Y, Z): 1 \leq l \leq k-1, X_{1}, \ldots, X_{l}, Y, Z \in T M\right\} \subseteq N M .
$$

By applying Proposition 2.1(iii) and Lemma 2.1(i) and using the Codazzi-Mainardi equation we can also write this as

$$
F_{0}=\operatorname{span}\left\{\left(\nabla_{X_{1}, \ldots, X_{l}}^{l} \alpha\right)(Y, Z): 1 \leq l \leq k-1, X_{1}, \ldots, X_{l}, Y, Z \in E_{0}\right\} \subseteq N M .
$$

Proposition 2.4. Let $(M, g)$ be a k-parallel isometric immersion into a space form. The following hold:

(i) the subbundle $F_{0} \subseteq N M$ is parallel w.r.t. the normal connection;

(ii) the bundle $F_{0}$ is flat, that is, $R^{\perp}(T M, T M) F_{0}=0$. 
Proof. (i) is a direct consequence of $\nabla^{k} \alpha=0$.

(ii) We use (2.6) for the description of $F_{0}$. Let $X_{1}, \ldots, X_{l}, Y, Z$ be in $E_{0}$ and $1 \leq l \leq k-1$. We have $R(U, V) \cdot \nabla^{l} \alpha=0$ for all $U, V \in T M$ by differentiating the equation $R(U, V) \cdot \alpha=0$, which we know from Proposition 2.2(i). This gives

$$
\begin{gathered}
R^{\perp}(U, V)\left(\left(\nabla_{X_{1}, \ldots, X_{l}}^{l} \alpha\right)(Y, Z)\right)=\sum_{j=1}^{l}\left(\nabla_{X_{1}, \ldots, R(U, V) X_{j}, \ldots, X_{l}}^{l} \alpha\right)(Y, Z) \\
+\left(\nabla_{X_{1}, \ldots, X_{l}}^{l} \alpha\right)(R(U, V) Y, Z)+\left(\nabla_{X_{1}, \ldots, X_{l}}^{l} \alpha\right)(Y, R(U, V) Z)
\end{gathered}
$$

Since $E_{0}$ is flat, the right-hand side of the above equation vanishes.

Lemma 2.2. If $(M, g)$ is a k-parallel isometric immersion into a space form, then

(i) $\alpha\left(E_{0}, E_{0}\right) \subseteq N_{0} M$;

(ii) $\alpha\left(E_{0}, E_{1}\right) \subseteq N_{1} M$;

(iii) $A_{N_{0} M} E_{i} \subseteq E_{i}, i=1,2$;

(iv) $A_{F_{0}} E_{1}=0$.

Proof. (i) Since $(M, g)$ is semiparallel by Proposition 2.2 and since $E_{0}$ is flat, we have

$$
R(U, V)^{\perp}(\alpha(X, Y))=\alpha(R(U, V) X, Y)+\alpha(X, R(U, V) Y)=0
$$

for all $X, Y \in E_{0}$ and all $U, V \in T M$.

(ii) Using again that $(M, g)$ is semiparallel and that $E_{0}$ is flat we obtain

$$
R^{\perp}(U, V)(\alpha(X, Y))=\alpha(R(U, V) X, Y)
$$

for all $U, V, X \in T M$ and $Y \in E_{0}$. The claim now follows from $E_{1}=\operatorname{span}\{R(X, Y) Z$ : $X, Y, Z \in T M\}$.

(iii) follows from (ii). Indeed,

$$
\left\langle A_{N_{0} M} E_{1}, E_{0}\right\rangle=\left\langle E_{1}, A_{N_{0} M} E_{0}\right\rangle=\left\langle\alpha\left(E_{1}, E_{0}\right), N_{0} M\right\rangle=0 .
$$

(iv) We have to show that $\alpha\left(E_{1}, T M\right) \perp F_{0}$. Obviously, $\alpha\left(E_{1}, E_{0}\right) \perp F_{0}$ by (ii) since Proposition 2.4(ii) gives $F_{0} \subset N_{0} M$. It remains to show that $\alpha\left(E_{1}, E_{1}\right) \perp F_{0}$. Take $Y_{0}, Z_{0} \in E_{0}$ and $Y_{1}, Z_{1} \in E_{1}$. Because $R\left(E_{0}, E_{1}, E_{0}, E_{1}\right)=0$ the Gauss equation (2.2) yields

$$
\left\langle\alpha\left(Y_{1}, Y_{0}\right), \alpha\left(Z_{1}, Z_{0}\right)\right\rangle-\left\langle\alpha\left(Y_{1}, Z_{1}\right), \alpha\left(Y_{0}, Z_{0}\right)\right\rangle=c\left\langle Y_{0}, Z_{0}\right\rangle\left\langle Y_{1}, Z_{1}\right\rangle .
$$

After differentiation, using that $\nabla_{E_{1}} \alpha=0$, the Codazzi-Mainardi equation leads to

$$
\left\langle\alpha\left(Y_{1}, Z_{1}\right),\left(\nabla_{X_{1}, \ldots, X_{l}}^{l} \alpha\right)\left(Y_{0}, Z_{0}\right)\right\rangle=0
$$

for all $1 \leq l \leq k-1$ and $X_{1}, \ldots, X_{l} \in E_{0}$. Now it follows from (2.6) that $\alpha\left(E_{1}, E_{1}\right)$ is orthogonal to $F_{0}$.

Now we will define a further decomposition of $E_{0}$ into subbundles $E_{0}^{\prime} \oplus E_{0}^{\prime \prime}$ such that Moore's criterion applies to $D_{0}:=E_{1} \oplus E_{0}^{\prime}$ and $D_{1}:=E_{0}^{\prime \prime}$. Let $E_{0}^{\prime \prime}$ be spanned by elements of the form

$$
A_{\xi} X,\left(\nabla_{U_{1}, \ldots U_{l}}^{l} A\right)_{\xi} X, \quad l=1, \ldots, k-1,
$$

where $\xi$ belongs to $F_{0}, X$ is in $T M$ and $U_{1}, \ldots, U_{l}$ are in $T M$. Equivalently, it suffices to take $X \in E_{0}$. Indeed, this follows from Lemma 2.2(iv).

Obviously, $E_{0}^{\prime \prime} \subset E_{0}$ by Lemma 2.2 (iii). We will denote by $E_{0}^{\prime}$ the orthogonal complement of $E_{0}^{\prime \prime}$ in $E_{0}$. 
Proposition 2.5. If $(M, g)$ is a k-parallel isometric immersion into a space form, then

(i) $E_{0}^{\prime}$ and $E_{0}^{\prime \prime}$ are parallel w.r.t. $\nabla$;

(ii) $E_{0}^{\prime} \subseteq\left\{X \in E_{0}: A_{F_{0}} X=0\right\}$;

(iii) $E_{0}^{\prime} \subseteq\left\{X \in E_{0}: \nabla_{X} \alpha=0\right\}$.

Proof. (i) follows directly from having $E_{0}$ and $F_{0}$ parallel and $\nabla^{k} \alpha=0$.

(ii) is a direct consequence of the definition of $E_{0}^{\prime}$.

(iii) Take $X \in E_{0}^{\prime}$. We first note that $\left(\nabla_{U} A\right)_{\xi} X=0$; hence also $\left\langle\left(\nabla_{U} \alpha\right)(X, \cdot), \xi\right\rangle$ $=0$ holds for all $U \in T M$ and $\xi \in F_{0}$ by the definition of $E_{0}^{\prime}$. By the CodazziMainardi equation we obtain $\left\langle\left(\nabla_{X} \alpha\right)(U, \cdot), \xi\right\rangle=0$ for all $U \in T M$ and $\xi \in F_{0}$. Since $\left(\nabla_{X} \alpha\right)(T M, T M) \subseteq F_{0}$ the claim follows.

Proposition 2.6. $\alpha\left(E_{1} \oplus E_{0}^{\prime}, E_{0}^{\prime \prime}\right)=0$.

Proof. The orthogonal complement $F_{1}$ of $F_{0}$ clearly contains $N_{1} M$. Take $\xi_{1} \in F_{1}$. Then

$$
\left\langle\left(\nabla_{U_{1}, \ldots, U_{l}}^{l} A\right)_{\xi_{1}} X, Y\right\rangle=\left\langle\left(\nabla_{U_{1}, \ldots, U_{l}}^{l} \alpha\right)(X, Y), \xi_{1}\right\rangle=0
$$

holds for all $X, Y, U_{1}, \ldots, U_{l} \in T M$ since $\left(\nabla_{U_{1}, \ldots, U_{l}}^{l} \alpha\right)(X, Y) \in F_{0}$. Thus

$$
\left(\nabla_{U_{1}, \ldots, U_{l}}^{l} A\right)_{\xi_{1}}=0 \text {. }
$$

The Ricci equation (2.5) gives

$$
\left[A_{\xi}, A_{\xi_{1}}\right]=0
$$

for all $\xi \in N_{0} M$ and $\xi_{1} \in F_{1}$, which implies that

$$
A_{\xi_{1}}\left(\operatorname{Im} A_{\xi}\right) \subseteq \operatorname{Im} A_{\xi} .
$$

Differentiating (2.8) and taking into account (2.7) we obtain

$$
\left[\left(\nabla_{U_{1}, \ldots, U_{l}}^{l} A\right)_{\xi}, A_{\xi_{1}}\right]=0 .
$$

This gives

$$
A_{\xi_{1}}\left(\operatorname{Im}\left(\nabla_{U_{1}, \ldots, U_{l}}^{l} A\right)_{\xi}\right) \subseteq \operatorname{Im}\left(\nabla_{U_{1}, \ldots, U_{l}}^{l} A\right)_{\xi}
$$

for all $U_{1}, \ldots, U_{l} \in T M$. Applying (2.9) and (2.10) to all $\xi \in F_{0}$ we obtain

$$
A_{F_{1}}\left(E_{0}^{\prime \prime}\right) \subseteq E_{0}^{\prime \prime} \text {. }
$$

Since $N_{1} M \subseteq F_{1}$ this implies that

$$
\left\langle\alpha\left(E_{0}^{\prime \prime}, E_{1}\right), N_{1} M\right\rangle=\left\langle A_{N_{1} M} E_{0}^{\prime \prime}, E_{1}\right\rangle=0,
$$

which combined with $\alpha\left(E_{0}, E_{1}\right) \subseteq N_{1} M$ shows that $\alpha\left(E_{0}^{\prime \prime}, E_{1}\right)=0$.

At the same time from (2.11),

$$
\left\langle\alpha\left(E_{0}^{\prime}, E_{0}^{\prime \prime}\right), F_{1}\right\rangle=\left\langle A_{F_{1}} E_{0}^{\prime \prime}, E_{0}^{\prime}\right\rangle=0,
$$

which shows that $\alpha\left(E_{0}^{\prime}, E_{0}^{\prime \prime}\right)$ is contained in $F_{0}$. However, $\left\langle\alpha\left(E_{0}^{\prime}, E_{0}^{\prime \prime}\right), F_{0}\right\rangle=$ $\left\langle A_{F_{0}} E_{0}^{\prime}, E_{0}^{\prime \prime}\right\rangle=0$ by (ii) in Proposition 2.5 leads to $\alpha\left(E_{0}^{\prime}, E_{0}^{\prime \prime}\right)=0$.

Proof of Theorem 2.3. The orthogonal splitting $T M=\left(E_{1} \oplus E_{0}^{\prime}\right) \oplus E_{0}^{\prime \prime}$ is parallel w.r.t. to the Levi-Civita connection and hence induces a local splitting of $M$ with corresponding factors $M_{1}$ and $M_{2}$. By Proposition 2.6. Molzan's generalisation of Moore's Theorem applies to the immersion of $M_{1} \times M_{2}$ in $M^{n}(c)$; it yields a product decomposition of $f$ according to Definition 2.2 with factors $f_{i}: M_{i} \rightarrow N_{i}, i=1,2$. 
From (iii) in Propositions 2.1 and 2.5 it follows that $\left(\varphi_{1} \circ f_{1}\right)\left(M_{1}\right)$ is parallel in $M^{n}(c)$. Since the image of $N_{1}$ in $M^{n}(c)$ is spherical, it follows by a standard argument that $\left(\varphi_{1} \circ f_{1}\right)\left(M_{1}\right)$ is parallel in $\varphi_{1}\left(N_{1}\right)$ as well. Hence $f_{1}: M_{1} \rightarrow N_{1}$ is parallel since $\varphi_{1}: N_{1} \rightarrow \varphi_{1}\left(N_{1}\right)$ is an isometry.

Since the distributions $E_{0}$ and hence $E_{0}^{\prime \prime}$ are flat, so is $M_{2}$ and the same argument as above shows that it is immersed in $N_{2}$ as a $k$-parallel immersion.

In the absence of a factor of type $E_{1}$ it follows from (ii) in Lemma 2.1 that the normal bundle of $M_{2}$ is flat.

\section{ACKNOWLEDGEMENT}

It is a pleasure to thank A. J. di Scala for useful comments on an earlier version of this paper.

\section{REFERENCES}

[1] E. Backes and H. Reckziegel, On symmetric submanifolds of spaces of constant curvature, Math. Ann. 263 (1983), 419-433. MR707240 (84i:53050)

[2] F. Dillen, The classification of hypersurfaces of a Euclidean space with parallel higher order fundamental form, Math. Z. 203 (1990), 635-643. MR1044069 (91d:53076)

[3] F. Dillen and S. Nölker, Semi-parallelity, multi-rotation surfaces and the helix-property, J. Reine Angew. Math. 435 (1993), 33-63. MR1203910 (94d:53083)

[4] N. Ejiri, Minimal immersions of Riemannian products into real space forms, Tokyo J. Math. vol. 2, no. 1, 1979. MR541897 (80h:53059)

[5] D. Ferus, Produkt-Zerlegung von Immersionen mit paralleler zweiter Fundamentalform, Math. Ann. 211 (1974), 1-5. MR0367876 (51:4118)

[6] D. Ferus, Immersions with parallel second fundamental form, Math. Z. 140 (1974), 87-92. MR.0370437 (51:6664)

[7] D. Ferus, Symmetric Submanifolds of Euclidean Space, Math. Ann. 247 (1980), 81-93. MR.565140(81i:53040)

[8] Ü. Lumiste, Small-dimensional irreducible submanifolds with parallel third fundamental form (Russian, summary in English), Tartu Ülik. Toim. Acta Comm. Univ. Tartuensis 734 (1986), 50-62. MR851351 (87k:53040)

[9] Ü.Lumiste, Submanifolds with a Van der Waerden-Bortolotti plane connection and parallelism of the third fundamental form, Izv. Vyssh. Uchebn. Zaved. Mat. 31 (1987), 18-27. MR.891995(88e:53033)

[10] Ü. Lumiste, Normally flat submanifolds with parallel third fundamental form, Proc. Estonian Acad. Sci. Phys. Math. 38, no. 2 (1989), 129-138. MR1046139 (91b:53022)

[11] Ü.Lumiste, Three dimensional submanifolds with parallel third fundamental form in Euclidean spaces, Tartu Ülikooli Toimetised. Acta et Comm. Univ. Tartuensis 889 (1990), 45-56. MR.1082922 (92c:53013)

[12] Ü. Lumiste, Submanifolds with parallel fundamental form, in F. Dillen and L. Verstraelen, eds., Handbook of Differential Geometry, Vol. I, Elsevier Science, Amsterdam, 2000, 779-864. MR,1736858 (2000j:53071)

[13] Ü.Lumiste, On submanifolds with parallel higher order fundamental form in Euclidean spaces, in B. Wegner, D. Ferus, U. Pinkall, and U. Simon, eds., Global Differential Geometry and Global Analysis, Lecture Notes in Mathematics, Vol. 1481, Springer-Verlag, Berlin, New York, 1991, 126-137. MR.1178526 (93i:53054)

[14] Ü.Lumiste, Semiparallel Submanifolds in Space Forms, Springer, 2008. MR2454627 (2010h:53080)

[15] V. A. Mirzojan, Submanifolds with a higher order parallel fundamental form (Russian), Akad. Nauk Armjan. SSR Dokl. 66 (1978), no. 2, 71-75. MR0487907 (58:7499)

[16] R. Molzan, Extrinsische Produkte und symmetrische Untermannigfaltigkeiten in Standardräumen konstanter und konstanter holomorpher Krümmung, Dissertation, Köln, 1983.

[17] J. D. Moore, Isometric immersions of riemannian products, J. Differential Geometry 5 (1971), 159-168. MR0307128 (46:6249) 
[18] O. I. Mokhov, Nonlocal Hamiltonian operators of hydrodynamic type with flat metrics, integrable hierarchies and the equations of associativity, Funct. Anal. Appl. 40 (2006), no. 1, 1-23. MR2223246 (2007b:37147)

[19] S. Nölker, Isometric immersions of warped products, Diff. Geom. Appl. 6 (1996), 1-30. MR:1384876(97d:53064)

[20] K. Nomizu and H. Ozeki, A theorem on curvature tensor fields, Proc. Nat. Acad. Sci. 48 (1962), 206-207. MR0132507 (24:A2347)

[21] W.Strübing, Symmetric submanifolds of Riemannian manifolds, Math. Ann. 245 (1979), 37-44. MR552577 (81a:53027)

[22] M. Takeuchi, Parallel submanifolds of space forms, in Manifolds and Lie Groups, Papers in Honor of Y. Matsushima, Progr. Math. 14 Birkhäuser, Boston, MA, 1981, 429-447. MR642871(83h:53078)

[23] S. Tanno, Curvature tensors and covariant derivatives. Ann. Mat. Pura Appl. (4) 96 (1972), 233-241. MR 0326619(48:4962)

Institut für Mathematik und Informatik, ERnSt-Moritz-Arndt Universität GreifsWald, Walther-Rathenau str. 47, 17487 Greifswald, Germany

E-mail address: ines.kath@uni-greifswald.de

Institut für Mathematik und Informatik, Ernst-Moritz-Arndt Universität Greifswald, Walther-Rathenau str. 47, 17487 Greifswald, Germany

E-mail address: nagyp@uni-greifswald.de 